\title{
A Flattened Protostellar Envelope in Absorption around L1157
}

\author{
Leslie W. Looney ${ }^{1}$, John J. Tobin ${ }^{2}$, and Woojin Kwon ${ }^{1}$
}

\begin{abstract}
Deep Spitzer IRAC images of L1157 reveal many of the details of the outflow and the circumstellar environment of this Class 0 protostar. In IRAC band $4,8 \mu \mathrm{m}$, there is a flattened structure seen in absorption against the background emission. The structure is perpendicular to the outflow and is extended to a diameter of $\sim 2^{\prime}$. This structure is the first clear detection of a flattened circumstellar envelope or pseudo-disk around a Class 0 protostar. Such a flattened morphology is an expected outcome for many collapse theories that include magnetic fields or rotation. We construct an extinction model for a power-law density profile, but we do not constrain the density power-law index.
\end{abstract}

Subject headings: stars: formation; stars: circumstellar matter; stars: pre-main sequence; infrared: stars

\section{Introduction}

The L1157 dark cloud in Cepheus (IRAS 20386+6751) conceals a young protostar, a so-called Class 0 source (Andre et al. 1993), which is deeply embedded within a large circumstellar envelope (Gueth et al. 2003; Beltrán et al. 2004). L1157 has a large powerful molecular outflow that is the prototype of chemically active outflows (Bachiller et al. 2001). Despite the attention that L1157 has received at radio wavelengths, few observations have been made in the near to mid-infrared outside of observations of the outflow in $\mathrm{H}_{2}$ and K-band (e.g. Davis \& Eisloeffel 1995; Cabrit et al. 1998). Only recently have sensitive instruments been available to observe these objects shortward of $10 \mu \mathrm{m}$ (e.g., Tobin et al. 2007). The outflow carves cavities in the circumstellar envelope, which allow photons from the embedded central source to escape and scatter off dust in the cavity at NIR wavelengths. The morphology of the scattered light can be used to probe many of the fundamental properties of the source such as opening angle, envelope mass, etc. (e.g., Whitney et al. 2003b,a; Tobin et al. 2007; Robitaille et al. 2007; Seale \& Looney 2008).

In this letter, we present new, deep Spitzer Space Telescope observations of L1157. The IRAC continuum emission is dominated by molecular line emission in the outflow. Near the source there

\footnotetext{
${ }^{1}$ Department of Astronomy, University of Illinois, 1002 W. Green St., Urbana, IL 61801, lwl@uiuc.edu, wkwon@uiuc.edu

${ }^{2}$ Department of Astronomy, University of Michigan, 500 Church St., Ann Arbor, MI 48108, jjtobin@umich.edu
} 
is a small amount of emission that may be attributed to scattered light and perhaps molecular line emission that is highly excited by the outflow jet. Other than the enormous outflow $(\sim 0.5 \mathrm{pc})$ to the north and south, the most prominent feature observed is a large, flattened absorption feature at $8.0 \mu \mathrm{m}$ and less defined at $5.8 \mu \mathrm{m}$. This absorption feature is a flattened circumstellar envelope observed in silhouette against the Galactic infrared background.

The distance to L1157 is important to any physical interpretation, but the distance is highly uncertain. The molecular clouds in Cepheus have three characteristic distances, 200, 300, 450 pc Kun 1998); L1157 has a similar galactic latitude as the 200 pc and 300 pc absorbing clouds. Due to this, we disagree with the current accepted distance of $440 \mathrm{pc}$. This value was based upon a study of NGC 7023 in Viotti (1969); L1157 is not in clear association with this cluster. In this letter, we use a distance of $250 \mathrm{pc}$.

\section{Observations}

The biggest breakthrough in observing Class 0 sources in scattered light has come with the sensitivity of the Infrared Array Camera (IRAC) (Fazio et al. 2004) on the Spitzer Space Telescope (Werner et al. 2004). This enables observers to see through the dust enshrouding a Class 0 source and reveal structures close to the embedded source via scattered light and emission lines. IRAC has channels numbered 1 through 4, corresponding to central wavelengths of $3.6 \mu \mathrm{m}, 4.5 \mu \mathrm{m}, 5.8$ $\mu \mathrm{m}$, and $8.0 \mu \mathrm{m}$, respectively.

The L1157 cloud was observed with IRAC on 2006 August 13. Observations were carried out using the High Dynamic Range mode with frame times of 30 seconds using a cycled dither pattern of 30 positions and a small scale factor achieving a total integration time of 900 seconds. This observing scheme increases the overall sensitivity to scattered light in the outflow cavity of Class 0 sources (e.g., Tobin et al. 2007). Post-BCD pipeline products (version S14.4.0) were solely used in this letter. A color-composite image, with exaggerated band 4 stretch of the field is shown in Figure 1. In addition, a greyscale image using only IRAC channel 4, $8 \mu \mathrm{m}$ band, is shown in Figure 2 .

\section{Results}

\subsection{Flattened Envelope in Absorption}

Even a cursory examination of Figures 1 and 2 reveals a clear absorption feature perpendicular

to the outflow. Much like the proplyds in Orion (e.g., O'dell et al. 1993), the structure is seen in absorption against a bright background. In our case, the bright and pervasive background emission is from the $8.0 \mu \mathrm{m}$ band. There is also a lower level of intrinsic background emission in the $5.8 \mu \mathrm{m}$ channel, which shows a less prominent absorption feature. However, the level of absorption is too 
low for a rigorous analysis. If the absorption is due to the opacity of the circumstellar material, we would also expect to see absorption features in the 3.6 and $4.5 \mu \mathrm{m}$, but in those channels, the background radiation field is nearly zero. The most likely explanation is that the background emission is from Polycyclic Aromatic Hydrocarbon (PAH) features, which are strongest in the 5.8 and $8 \mu \mathrm{m}$ bands (e.g., Fazio et al. 2004). PAHs are typically fluorescing due to the absorption of ultraviolet photons from the surrounding interstellar radiation field; thus, PAH emission is a ubiquitous feature of the diffuse interstellar medium (e.g., Flagev et al. 2006). So while the proplyds in Orion are seen against the bright nebula emission, L1157 is seen against PAH emission. The L1157 absorption feature is large, $\sim 1-2^{\prime}$ or $\sim 15,000-30,000$ AU at a distance of 250 pc (see \$1) and flattened with an axis ratio of $\sim 0.25$. Due to the size and lack of any kinematic information, we will call it a flattened envelope or a pseudo-disk, not a circumstellar disk; such flattened density enhancements are expected from many theoretical constructs that include magnetic fields or rotation in the collapse process (e.g., Terebey et al. 1984; Fiedler \& Mouschovias 1993; Galli \& Shu 1993a, b; Hartmann et al. 1994, 1996).

The observed extinction is probably dominated by a combination of silicates and ice. The $8.0 \mu \mathrm{m}$ channel overlaps with the $9.7 \mu \mathrm{m}$ silicate feature in half of the bandpass. However, ice features are present in both the 5.8 and $8.0 \mu \mathrm{m}$ channels: the $6.85 \mu \mathrm{m}\left(\mathrm{CH}_{3} \mathrm{OH}\right.$ or $\left.\mathrm{NH}_{4}^{-}\right)$and the $9.0 \mu \mathrm{m} \mathrm{NH}$ ice features in the $8.0 \mu \mathrm{m}$ channel, and the $6.0 \mu \mathrm{m} \mathrm{H}_{2} \mathrm{O}$ ice feature in the $5.8 \mu \mathrm{m}$ channel. The qualitative appearance of the absorption does correlate very well with observations of the dust continuum emission structure seen at $\lambda=1.3 \mathrm{~mm}$ (Gueth et al. 2003), as well as the ammonia emission Bachiller et al. (1993), but as these trace the dense core, this may not be especially surprising.

\subsection{Outflow Features}

L1157 has one of the most studied and well-developed bipolar outflows. The outflow has an inclination of $\sim 80^{\circ}$ and a slower blueshifted (southern) lobe than the redshifted (northern) lobe (e.g. Gueth et al. 1996; Bachiller et al. 2001). The S shape morphology and the three peaks at point reflection symmetry seen in $\mathrm{CO}$ and $\mathrm{SiO}$ emission are well explained by outflow precession with a cone angle of $\sim 15^{\circ}$ (Gueth et al. 1996; Zhang et al. 2000; Bachiller et al. 2001).

The IRAC data have a remarkable coincidence of emission structure with the molecular outflows, Figure 2. We measure the largest separation of peaks as $\sim 15^{\circ}$ and an angle of the outflow extension in width ("east-west" direction) as $\sim 35^{\circ}$. This means that the precession cone has a $\sim 15^{\circ}$ angle, consistent with previous studies (e.g. Zhang et al. 2000; Bachiller et al. 2001), and that each episodic shock has $\sim 10^{\circ}$ opening angle. The precession cone angle here indicates the total angle of the cone, and the opening angle represents half of the outflow opening. From the peak positions, the precession period is estimated as $\sim 3050$ years, assuming $250 \mathrm{pc}$ distance and $100 \mathrm{~km} \mathrm{~s}^{-1}$ constant outflow velocity from the model of Bachiller et al. (2001). 
Unlike the extinction structure, the outflow features are commonly shown in all four IRAC bands. The excitation mechanism for the broad energy range of emission is beyond the scope of this letter; it requires spectroscopic observations and detailed modeling to understand level populations in a large energy region. However, we can consider hydrogen recombination lines (the bipolar outflow regions were dissociatively shocked, e.g., Bachiller et al. 2001), and molecular hydrogen ro-vibrational and rotational lines (based on the chemical complexity of the outflow). In fact, many of these transitions are in the IRAC bands with some metal ion fine structure lines (e.g. Rosenthal et al. 2000; Noriega-Crespo et al. 2004; Neufeld et al. 2006). The $3.6 \mu \mathrm{m}$ band may be dominated by $\mathrm{H}_{2} 1-0 \mathrm{O}(5,6,7)$ and $0-0 \mathrm{~S}(13)$. IRAC bands 2,3 , and 4 may be dominated by $\mathrm{H}_{2}$ 0-0 $\mathrm{S}(12,11,10,9), \mathrm{S}(8,7,6)$, and $\mathrm{S}(5,4)$, respectively. Some hydrogen recombination lines such as Brackett $\alpha$ in band 2 and ion fine structures such as [Ni II], [Ar II], and [Ar III] in band 4 also may contribute. Finally, PAH emission contributes in bands 3 and 4.

\subsection{Optical Depth Measurement and Models}

The absorption feature of the flattened envelope structure in L1157 provides an excellent opportunity to examine the envelope material by measuring the optical depth along the feature. This is especially true as the L1157 flattened envelope is nearly edge-on, isolated, and surrounded by relatively smooth background emission. The background and associated average uncertainty was measured using the IRAF 1 task "imstat" in three areas within $90^{\prime \prime}$ from the central source $(0.54 \pm$ $0.04 \mathrm{MJy} / \mathrm{sr}$ ). The areas were free of stars and not significantly affected by residual absorption from the outer envelope. A constant background may not be entirely realistic, but we did explore the possibility of modeling the background using median filtering in a similar method to Simon et al. (2006). However, using a background model did not improve the analysis.

The intensity of the absorption feature was measured perpendicular to the outflow, radially away from the central source. This was done using SAOImage DS9 to measure a "projection" of 82 pixels in length and 3 pixels in width. The intensity was taken as the average of the 3 pixels (in width) for a particular position along the projection. To determine the intensity uncertainty, we used the pixel values provided by the uncertainty frame from the Spitzer pipeline. Note, that the point spread function of IRAC at $8.0 \mu \mathrm{m}$ is $1^{\prime \prime} .9$, and the pixel size is $1^{\prime \prime} .2$ (Fazio et al. 2004).

In order to quantitatively compare the absorption to axisymmetric models of circumstellar envelopes, we created a simple model of the opacity through an absorption slice, perpendicular to the outflow. The model consists of an edge-on flattened object, i.e. disk-like, with a radial density profile, $\rho(r)=\rho_{0}\left(r / r_{0}\right)^{-p}$, where $r_{0}$ and $\rho_{0}$ are the radius and the density one pixel away from the center. Using a Cartesian convention where location along the absorption feature is $x$ and the

\footnotetext{
${ }^{1}$ IRAF is distributed by the National Optical Astronomy Observatories, which are operated by the Association of Universities for Research in Astronomy, Inc., under cooperative agreement with the National Science Foundation.
} 
distance along the line of sight is $y$, one can re-write the density profile along the absorption as $\rho(x, y)=\rho_{0}\left(\left(x^{2}+y^{2}\right) / r_{0}^{2}\right)^{-p / 2}$ The opacity is then of the form

$$
\tau(x)=2 \kappa_{\lambda} \rho_{0} r_{0} \int_{0}^{\sqrt{R^{2}-x^{2}}}\left(\left(x^{2}+y^{2}\right) / r_{0}^{2}\right)^{-p / 2} d\left(y / r_{0}\right),
$$

where $\mathrm{R}$ is the outer radius of the disk and $\kappa_{\lambda}$ is the dust opacity. This is compared to the observed opacity of $\tau(x)=-\ln ($ intensity $(\mathrm{x}) /$ background) in the average along the projection. This is an edge-on approximation; the source has an $\sim 80^{\circ}$ inclination, but as we are averaging over three vertical pixels, the effect is minimized.

To fit this model to the data, a grid of models was used for the following variables: the background level (although we measure a background level, we still use a grid of values around the measured value), a constant for $\kappa_{\lambda}$ and $\rho_{0}$ combined, the outer disk radius $(R)$, the location of the structure center in pixels $\left(X_{0}\right)$, and the power law dependence $(p)$. Note that there are no a priori assumptions as to the values of $\kappa_{\lambda}$ or $\rho_{0}$. This parameter space is then compared to the $8.0 \mu \mathrm{m}$ data using a $\chi^{2}$ likelihood. The pixels close to the central source were not used in the fits, as they contain bright emission from the scattered light close to the protostar, see Figure 3 ,

Our model parameter grid used background levels $=0.45$ to $0.60 \mathrm{MJy} / \mathrm{sr}$ in steps of 0.01 , $\mathrm{p}=0.5$ to 3 in steps of $0.5, R=18^{\prime \prime}$ to $84^{\prime \prime}$ in steps of $1.2^{\prime \prime}, X_{0}$ was fixed to $0^{\prime \prime}$ or $\pm 1.2^{\prime \prime}$ of the peak of the compact dust emission (Beltrán et al. 2004), and the constant=0.01 to 2.0 in steps of 0.01 . The model had nearly 3 million grid points. Using these parameters, the simple model of optical depth successfully fit the data with high confidence levels. Models were considered good fits if the confidence level was $>90 \%$, i.e. $>10 \%$ likelihood (see Table 1). In general, the fits are not well constrained. The power-law index, $p$, fits range from 0.5 to 2.0 , and the outer radius, $R$, was constrained to $\geq 27.6^{\prime \prime}$; however, the maximum is not well constrained as we only modeled $\pm 50^{\prime \prime}$ of data. Examples of the "best fits" for each acceptable density power-law are shown on the $8.0 \mu \mathrm{m}$ data in Figure 3 ,

\section{Discussion}

The shape of the absorption feature is especially intriguing, as it looks like a disk structure perpendicular to the outflow axis. This is the first clear detection of a flattened envelope or pseudodisk in a Class 0 object. Galli \& Shu (1993a, b) have shown that a modest magnetic field structure modifies infall from the initial spherical cloud to form a so-called "pseudo-disk"; a flat thin structure in the equatorial plane that is not rotationally-supported, thus collapsing. This type of structure is also seen from simple flat sheet models of collapse (e.g., Hartmann et al. 1994, 1996), as well as detailed ambipolar diffusion models (e.g., Fiedler \& Mouschovias 1993). On the other hand, this structure is large $\sim 15,000-30,000 \mathrm{AU}$, depending on the background used. That size is somewhat larger than the inner envelope size estimated from interferometric models of the dust continuum (Looney et al. 2003). However, the single-dish dust emission (Gueth et al. 2003) is extended along 
the same axis as the absorption, which argues that the inner envelope in the equatorial plane either has higher density, and/or different dust opacity properties.

Our modeling results show that the properties of the structure, as determined by the absorption model, are consistent with the above theoretical constructs, i.e. flattened envelopes and density profiles. Although we model a range of indexes ranging from $\mathrm{p}=0.5$ to 3 , only the 0.5 to 2 provide acceptable fits at the $90 \%$ confidence level with the vast majority of fits being $\mathrm{p}=1.5$.

To better explore the physical meaning the model, we assume dust opacities (dust plus gas) from Li \& Draine (2001) of $\kappa_{8.0 \mu m}=5.912 \mathrm{~cm}^{2} \mathrm{~g}^{-1}$. Although using interstellar dust opacities for $\kappa_{\lambda}$ is probably incorrect, as Class 0 sources are thought to have already experienced some grain growth (e.g., Looney et al. 2003; Natta et al. 2007), it is still a useful approximation. Using the assumed $\kappa_{8.0 \mu m}$, the derived range for the density reference, $\rho_{0}$, or 1 pixel $\left(1^{\prime \prime} 2\right)$ from the center of the envelope (i.e. $300 \mathrm{AU}$ at $250 \mathrm{pc}$ ) is listed in Table 1.

In addition, we can estimate the absorbing mass of the flattened envelope component for each "best fit" model of Figure 3 and a height of 3 pixels (the size of the box we averaged over). We assume that the vertical density profile is constant for the mass estimate, even though the observed absorption falls off vertically with scale heights of $\sim 3-4$ pixels, using a Gaussian vertical structure. Our mass estimate, listed in Table1, ranges from 0.08 to $0.16 \mathrm{M}_{\odot}$. Without using any model, we can also estimate the mass required for the observed extinction. We use the above value for $\kappa_{8.0 \mu m}$ and a background of $0.54 \mathrm{MJy} / \mathrm{sr}$ to calculate the mass necessary for the extinction of all pixels in the central region below $0.458 \mathrm{MJy} / \mathrm{sr}$ (577 pixels). The total absorbing mass required for those pixels is $0.19 \mathrm{M}_{\odot}$. It is important to note that this mass is only in the absorbing pseudo-disk, but the mass is comparable to the $140^{\prime \prime}$ extended envelope detected in the millimeter continuum as discussed in Gueth et al. (2003) with an estimated mass of $0.7 \mathrm{M}_{\odot}$ at an assumed distance of $250 \mathrm{pc}$. This implies that although a large fraction of the mass of the envelope is in the flattened structure, most of the mass is more diffuse. Due to the log nature of the mass absorption, the density contrast from the center of the absorption feature to slightly offset from the absorption feature is approximately an order of magnitude. This is more than expected from the numerical models of Galli \& Shu (1993b), but somewhat consistent with the ambipolar models of Fiedler \& Mouschovias (1993) and the sheet collapse model of Hartmann et al. (1996).

As can be seen in Table 1 and Figure 3, these data can not constrain the model density profiles directly; multiple power-laws are allowed. On the other hand, although many theoretical models do suggest a flattened envelope structure, the power-law index in the pseudo-disk is expected to evolve. In that case, we would not expect a single power-law to well describe the envelope density. In fact, the ambipolar models (e.g. Tassis \& Mouschovias 2005a, $\underline{\text { b) }}$ ) suggest that the power-law can be episodic in the flattened envelope. Further studies with increased sensitivity need to be compared directly to the theoretical density profiles in the flattened objects to say anything more completely.

We thank F. Gueth for providing the $\lambda=1.3 \mathrm{~mm}$ continuum emission map and R. Bachiller for 
providing the CO 2-1 emission map. This work is based on observations made with the Spitzer Space Telescope, which is operated by the Jet Propulsion Laboratory, California Institute of Technology under a contract with NASA. Support for this work was provided by NASA. This research has made use of SAOImage DS9, developed by Smithsonian Astrophysical Observatory

\section{REFERENCES}

Andre, P., Ward-Thompson, D., \& Barsony, M. 1993, ApJ, 406, 122

Bachiller, R., Martin-Pintado, J., \& Fuente, A. 1993, ApJ, 417, L45+

Bachiller, R. \& Perez Gutierrez, M. 1997, ApJ, 487, L93+

Bachiller, R., Pérez Gutiérrez, M., Kumar, M. S. N., \& Tafalla, M. 2001, A\&A, 372, 899

Beltrán, M. T., Gueth, F., Guilloteau, S., \& Dutrey, A. 2004, A\&A, 416, 631

Cabrit, S. et al. 1998, in Astronomical Society of the Pacific Conference Series, Vol. 132, Star Formation with the Infrared Space Observatory, ed. J. Yun \& L. Liseau, 326-+

Davis, C. J. \& Eisloeffel, J. 1995, A\&A, 300, 851

Fazio, G. G. et al. 2004, ApJS, 154, 10

Fiedler, R. A. \& Mouschovias, T. C. 1993, ApJ, 415, 680

Flagey, N., Boulanger, F., Verstraete, L., Miville Deschênes, M. A., Noriega Crespo, A., \& Reach, W. T. 2006, A\&A, 453, 969

Galli, D. \& Shu, F. H. 1993a, ApJ, 417, 220

一. 1993b, ApJ, 417, 243

Gueth, F., Bachiller, R., \& Tafalla, M. 2003, A\&A, 401, L5

Gueth, F., Guilloteau, S., \& Bachiller, R. 1996, A\&A, 307, 891

Hartmann, L., Boss, A., Calvet, N., \& Whitney, B. 1994, ApJ, 430, L49

Hartmann, L., Calvet, N., \& Boss, A. 1996, ApJ, 464, 387

Kun, M. 1998, ApJS, 115, 59

Li, A. \& Draine, B. T. 2001, ApJ, 554, 778

Looney, L. W., Mundy, L. G., \& Welch, W. J. 2003, ApJ, 592, 255 
Natta, A., Testi, L., Calvet, N., Henning, T., Waters, R., \& Wilner, D. 2007, Protostars and Planets $\mathrm{V}, 767$

Neufeld, D. A., Melnick, G. J., Sonnentrucker, P., Bergin, E. A., Green, J. D., Kim, K. H., Watson, D. M., Forrest, W. J., \& Pipher, J. L. 2006, ApJ, 649, 816

Noriega-Crespo, A., Moro-Martin, A., Carey, S., Morris, P. W., Padgett, D. L., Latter, W. B., \& Muzerolle, J. 2004, ApJS, 154, 402

O'dell, C. R., Wen, Z., \& Hu, X. 1993, ApJ, 410, 696

Robitaille, T. P., Whitney, B. A., Indebetouw, R., \& Wood, K. 2007, ApJS, 169, 328

Rosenthal, D., Bertoldi, F., \& Drapatz, S. 2000, A\&A, 356, 705

Seale, J. P. \& Looney, L. W. 2008, ApJ, submitted

Simon, R., Jackson, J. M., Rathborne, J. M., \& Chambers, E. T. 2006, ApJ, 639, 227

Tassis, K. \& Mouschovias, T. C. 2005a, ApJ, 618, 769

—. 2005b, ApJ, 618, 783

Terebey, S., Shu, F. H., \& Cassen, P. 1984, ApJ, 286, 529

Tobin, J. J., Looney, L. W., Mundy, L. G., Kwon, W., \& Hamidouche, M. 2007, ApJ, 659, 1404

Viotti, R. 1969, Memorie della Societa Astronomica Italiana, 40, 75

Werner, M. W. et al. 2004, ApJS, 154, 1

Whitney, B. A., Wood, K., Bjorkman, J. E., \& Cohen, M. 2003a, ApJ, 598, 1079

Whitney, B. A., Wood, K., Bjorkman, J. E., \& Wolff, M. J. 2003b, ApJ, 591, 1049

Zhang, Q., Ho, P. T. P., \& Wright, M. C. H. 2000, AJ, 119, 1345 
Table 1. Summary of Acceptable Fits

\begin{tabular}{cccccccc}
\hline \hline $\begin{array}{c}\text { Power law } \\
\mathrm{p}\end{array}$ & $\begin{array}{c}\text { Background } \\
(\mathrm{MJy} / \mathrm{sr})\end{array}$ & $\begin{array}{c}R \\
\left({ }^{\prime \prime}\right)\end{array}$ & $\begin{array}{c}\text { Constant } \\
\left(\kappa_{\lambda} \rho_{0} r_{0}\right)\end{array}$ & $\begin{array}{c}\rho_{0}{ }^{\mathrm{a}} \\
\left(10^{-18} \mathrm{~g} / \mathrm{cm}^{3}\right)\end{array}$ & $\begin{array}{c}\text { Mass }{ }^{\mathrm{b}} \\
\left(\mathrm{M}_{\odot}\right)\end{array}$ & $\begin{array}{c}\text { Max. } \\
\text { prob. }(\%)\end{array}$ & $\begin{array}{c}\text { Number } \\
\text { of fits }\end{array}$ \\
\hline 0.5 & $0.47-0.60$ & $27.6-50.4$ & $0.02-0.03$ & $0.75-1.13$ & 0.08 & 99.2 & 130 \\
1.0 & $0.46-0.60$ & $28.8-84.0$ & $0.06-0.13$ & $2.26-4.90$ & 0.16 & 99.9 & 1820 \\
1.5 & $0.46-0.60$ & $30.0-84.0$ & $0.26-0.56$ & $9.80-21.11$ & 0.14 & 99.9 & 7253 \\
2.0 & $0.47-0.51$ & $34.8-84.0$ & $1.04-1.63$ & $39.20-61.43$ & 0.15 & 48.3 & 2622 \\
\hline
\end{tabular}

${ }^{a}$ Assuming $d=250 \mathrm{pc}$, for other distances multiply by (250/d).

${ }^{\mathrm{b}}$ The mass is estimated using the "best-fit" models from Figure 3 with $\mathrm{d}=250 \mathrm{pc}$. For other distances multiply by $(\mathrm{d} / 250)^{3}$. 


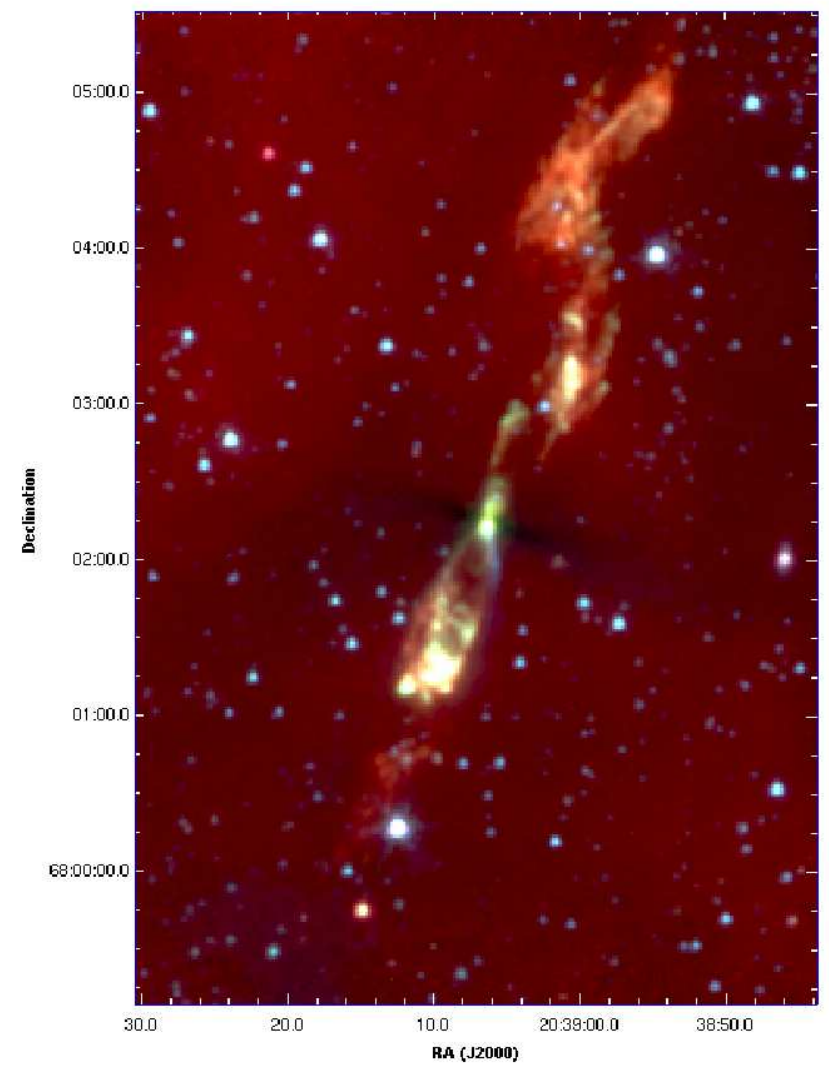

Fig. 1. - Color IRAC image of the L1157 region with Ch1-blue, Ch2-green, and Ch4-red. The color stretch is slightly exaggerated to emphasize Ch4 $(8 \mu \mathrm{m}$ band $)$ where the extinction is the largest. 


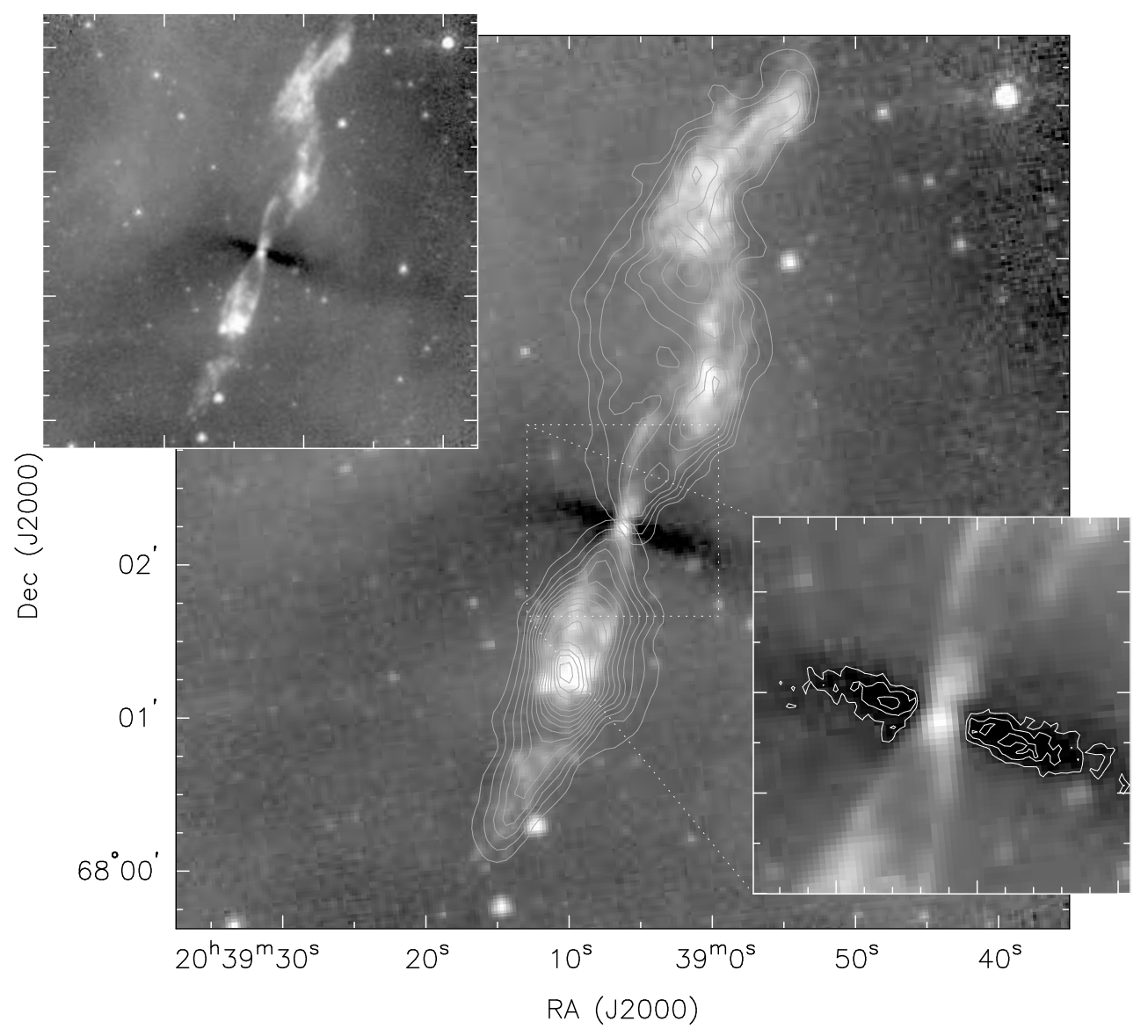

Fig. 2.- IRAC band 4 greyscale image of L1157 overlaid with the CO 2-1 emission from Bachiller et al. (2001). The upper-left inset is the same greyscale without the contours. The lower-right inset is a closeup $\left( \pm 75^{\prime \prime}\right)$ of the absorption structure with contours of $0.042 \mathrm{MJy} / \mathrm{sr} \times$ $1,2,3$, and 4 . 


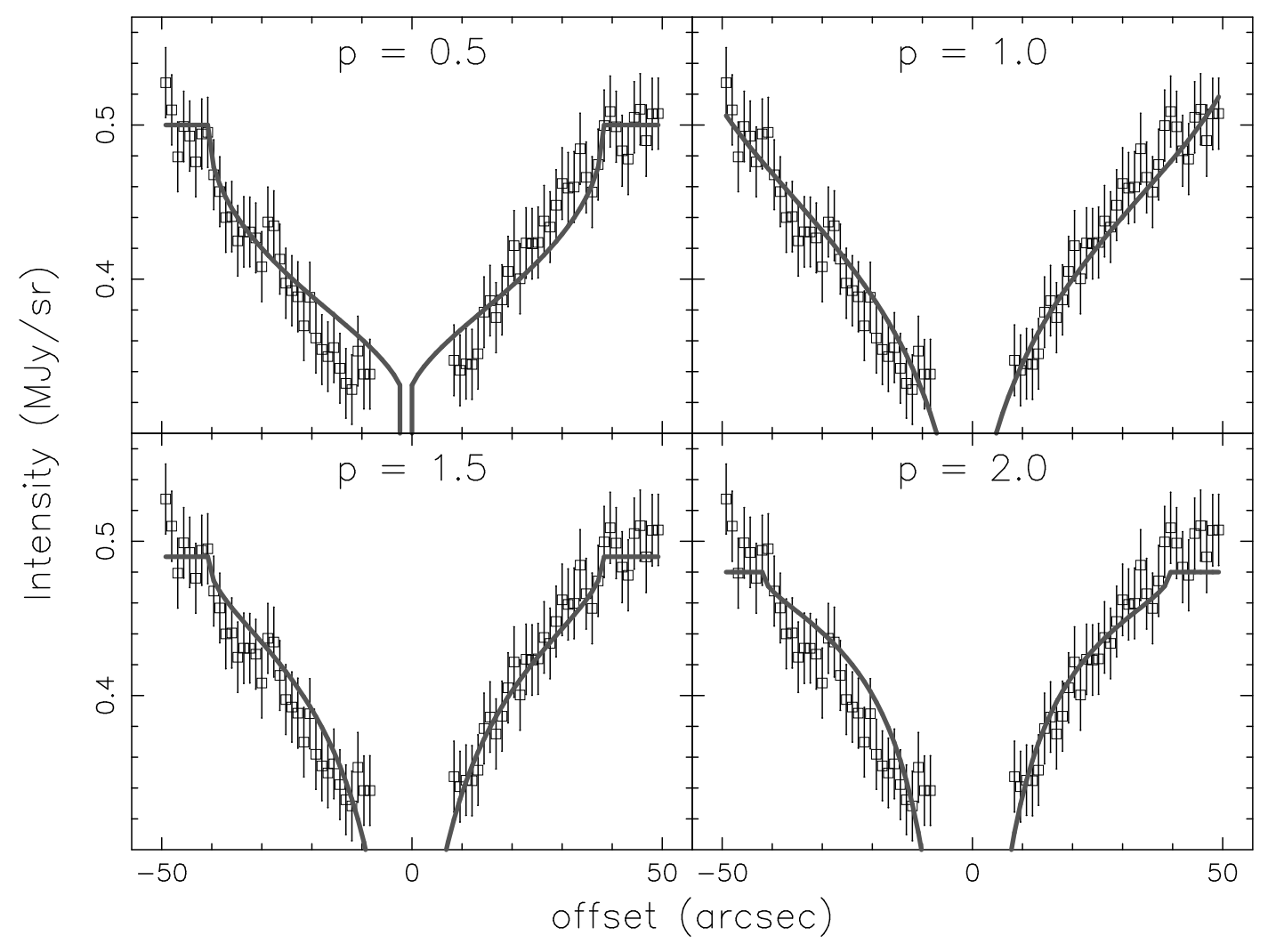

Fig. 3.- "Best fit" examples for the 4 acceptable density power-law indexes on the $8 \mu \mathrm{m}$ data. The fit parameters are: $(\mathrm{p}=0.5)$ background $=0.5 \mathrm{MJy} / \mathrm{sr}$, constant $=0.02, X_{0}=-1.2^{\prime \prime}$, and $\mathrm{R}$ $=39.6^{\prime \prime} ;(\mathrm{p}=1)$ background $=0.57 \mathrm{MJy} / \mathrm{sr}$, constant $=0.11, \mathrm{X}_{0}=-1.2^{\prime \prime}$, and $\mathrm{R}=55.2^{\prime \prime} ;(\mathrm{p}=1.5)$ background $=0.49 \mathrm{MJy} / \mathrm{sr}$, constant $=0.37, \mathrm{X}_{0}=-1.2^{\prime \prime}$, and $\mathrm{R}=55.2^{\prime \prime} ;(\mathrm{p}=2.0)$ background $=$ $0.48 \mathrm{MJy} / \mathrm{sr}$, constant $=1.3, \mathrm{X}_{0}=-1.2^{\prime \prime}$, and $\mathrm{R}=40.8^{\prime \prime}$. 